\title{
Optimising unit operations in paste dewatering
}

\author{
J. Palmer Outotec Pty Ltd, Australia \\ V. Viswanathan Outotec Pty Ltd, Australia
}

\begin{abstract}
Current practises in tailings handling are gradually shifting as there are increased pressures for water recovery and changing legislations for tailings impoundment. Many studies have been undertaken on paste thickening and tailings filtrations that have focused on a single unit operation.

This study investigates the interrelationships between gravity separation, particle separation, filtration, pumpability and materials handling in an attempt to optimise the process both from a process and operational expense (OPEX) perspective.

Paste thickeners can achieve high levels of water recovery yet their underflow is difficult to transport to disposal. High shear in the settling zones has significantly increased underflow densities but the discharge has a high viscosity that is at the limit of current pumping technologies and there is an issue with the distribution in the impoundment.
\end{abstract}

Filtration can achieve dry cake for solid disposal at the expense of high costs. Low feed densities and ultra fine particles can significantly impact on both the unit capacity and achievable moisture. More difficult slurries require increasingly higher pressures for separation and this substantially increases the separation costs.

Currently, tailings are a composite of all processing plants wastes and represent solids with a wide range of particle sizes, process water, excess reagents and spillage. Parts of these streams are relatively easy to dewater while others may be extremely difficult to handle. As a composite they tend to behave more like the worst component.

By changing the material flows and altering the duty points of filters and paste thickeners, superior performance can be achieved compared to either technology operating in isolation.

\section{Introduction}

\subsection{Water in mining}

Water is an essential component in most mineral processing applications. While some dry processes utilise size, magnetic or gravity separation exist, they are the minority and most applications where flotation or hydrometallurgy is employed to recover the valuable minerals utilise wet processing.

Water is first added in the milling stage and this and the associated classification or processing circuit determine the pulp density for the remainder of the process. Water recovery is necessary both because it is a scarce commodity and to minimise the tailings impoundment requirements.

Water recovery is usually accomplished through sedimentation or filtration processes. Water can be reduced from the tailings impoundment by evaporation but this does not improve the water consumption.

\subsection{Sedimentation}

Sedimentation is a process where a slurry or solid liquid mixture is separated to a solid rich and a liquid rich fraction in a force (gravitational) field due to the differences in phase densities. In mining applications sedimentation is applied to both the product and tailings streams to recover water which is recycled in the process. 
In a typical flotation concentrator the milling circuit is optimised with a pulp density of $25-35 \%$ solids w/w and with sedimentation to $60 \% \mathrm{w} / \mathrm{w}$ in a conventional tailings thickener $70 \%$ of the water required for processing can be recycled.

The degree of separation achievable with sedimentation is affected by the particle size shape, surface chemistry and solids density. These variables are evident in thickeners as the rise rate which determines the area required to separate the design slurry flow and the compression time which determines the depth.

By adding flocculants and increasing the depth of thickeners higher degrees of separation can be achieved. At low concentrations solid liquid mixtures behave like a liquid and at high concentrations they become a solid. In this slow transition from liquid to solid the pulp displays an exponential increase in yield stress and this translates to a limit to pumpability which in practise limits the degree of separation achievable.

Currently this limit is set at about $70 \% \mathrm{w} / \mathrm{w}$ for conventional thickening or $150 \mathrm{~Pa}$ for centrifugal pumping, which represents $82 \%$ recovery of process water.

\subsection{Filtration}

Filtration is a process where a slurry or solid liquid mixture is forced through a media with the solids retained on the media and the liquid phase passing through. In mining applications this slurry is generally either the product or mine tailings.

The product or concentrate is generally the smaller of the two streams where water removal is targeted to transportable limits and reducing transport costs. The transportable limit (TML) is a must for the product to be saleable and filtration technologies are selected based on their ability to reliably achieve this limit. The ability to reduce the moisture significantly below this limit may have limited effect on the product quality, transport cost or overall plant water balance.

The term TML is derived from the solid bulk cargoes industries where cargoes such as metal concentrates may appear to be in a relatively dry granular state when loaded, however, they may still contain sufficient moisture to become fluid under the stimulus of the compaction and vibration that occurs during a voyage. The resulting cargo shift can be sufficient to capsize the vessel.

To achieve this level where the product cannot become fluid it is necessary to pack or settle the solids to a point close to their incompressible limit and then further reduce the water by gas displacement. For example, a typical copper concentrate may have a TML of $9 \%$ moisture. Given that at the incompressible limit the cake will have a solids fraction of about $50 \% \mathrm{v} / \mathrm{v}$ with a typical particle size distribution and for solids with a $\mathrm{sg}$ of 4.3 , the moisture would be $18.9 \% \mathrm{w} / \mathrm{w}$ without gas displacement. The filtration process must displace about $50 \%$ of the remaining water with air to achieve the required moisture.

In extreme cases where the product is subject to freezing or oxidation lower moisture levels of $5 \%$ moisture may be required, where $75 \%$ of the internal water must be displaced. These levels are at the limit of the possibilities for water removal by filtration. Thermal drying is required if lower levels are required.

Tailings dewatering, in contrast to the product, is generally a much larger tonnage and the water removal objectives are targeted to maximise water recovery or disposal constraints. While water recovery may be important and in some cases can limit production generally the selection of filtration for tailings dewatering is based on the decision to apply dry stacking of the tailings.

Dewatering tailings produce a filtered wet cake that can no longer be transported by pipeline as it has lost fluid properties and is a solid. These filtered tailings are normally transported by conveyor or truck, deposited, spread and compacted to form an unsaturated tailings deposit.

At the incompressible limit, the cake will have a solids fraction of about $50 \% \mathrm{v} / \mathrm{v}$, for solids with a sg of 2.9 the cake moisture would be $25 \% \mathrm{w} / \mathrm{w}$ without gas displacement. The solids fraction varies depending upon the particles size, shape, distribution and method of filtration. For slurries with a composite of fines and coarse particles, a typical moisture content of less than $20 \%$ can be achieved by using filtration systems and with slurries low in fines moisture contents as low as $12 \% \mathrm{w} / \mathrm{w}$ can be achieved. 
At a typical cake with $80 \% \mathrm{w} / \mathrm{w}$ solids, almost $90 \%$ of the process water can be recycled. At the limit of presently available filtration systems capability, a cake of about $88 \% \mathrm{w} / \mathrm{w}$ can be achieved which represents a $94 \%$ recovery of process water.

\section{Sedimentation}

\subsection{Paste thickening}

Dewatering mineral slurries to form paste is presently one of the hottest topics in the minerals industry. The benefits derived from the creation of paste vary from improved throughput in autoclave circuits to environmentally sustainable tailings management via paste backfill systems. Flow sheets incorporating paste formation are easily derived and conceptually simple in that they combine well documented mineral processing steps such as thickening, filtration and slurry pumping.

Paste thickening technology is rapidly emerging as an effective method for handling tailings for water recovery and as an alternative to conventional dams and ponds. Paste and thickened tailings has been increasingly considered for tailings disposal. However, as with many things in mineral processing, the successful application of paste flow sheets to real plants is proving to be a challenge.

As shown in Figure 1, the consistency (solids content or density) of these tailings will also be limited by the capability of a centrifugal pump to drive the material through a pipeline. Positive displacement (PD) pumps will pump much higher density materials at correspondingly higher discharge pressures. The cost of installing and operating positive displacement pumps has to be evaluated over the lifetime of the project to make a meaningful comparison with a system using multiple centrifugal pumps (or pump stations) required to generate a comparable pump discharge pressure.

PASTE AND THICKENED TAILINGS

PROPERTIES AND DEFINITION OF TERMS

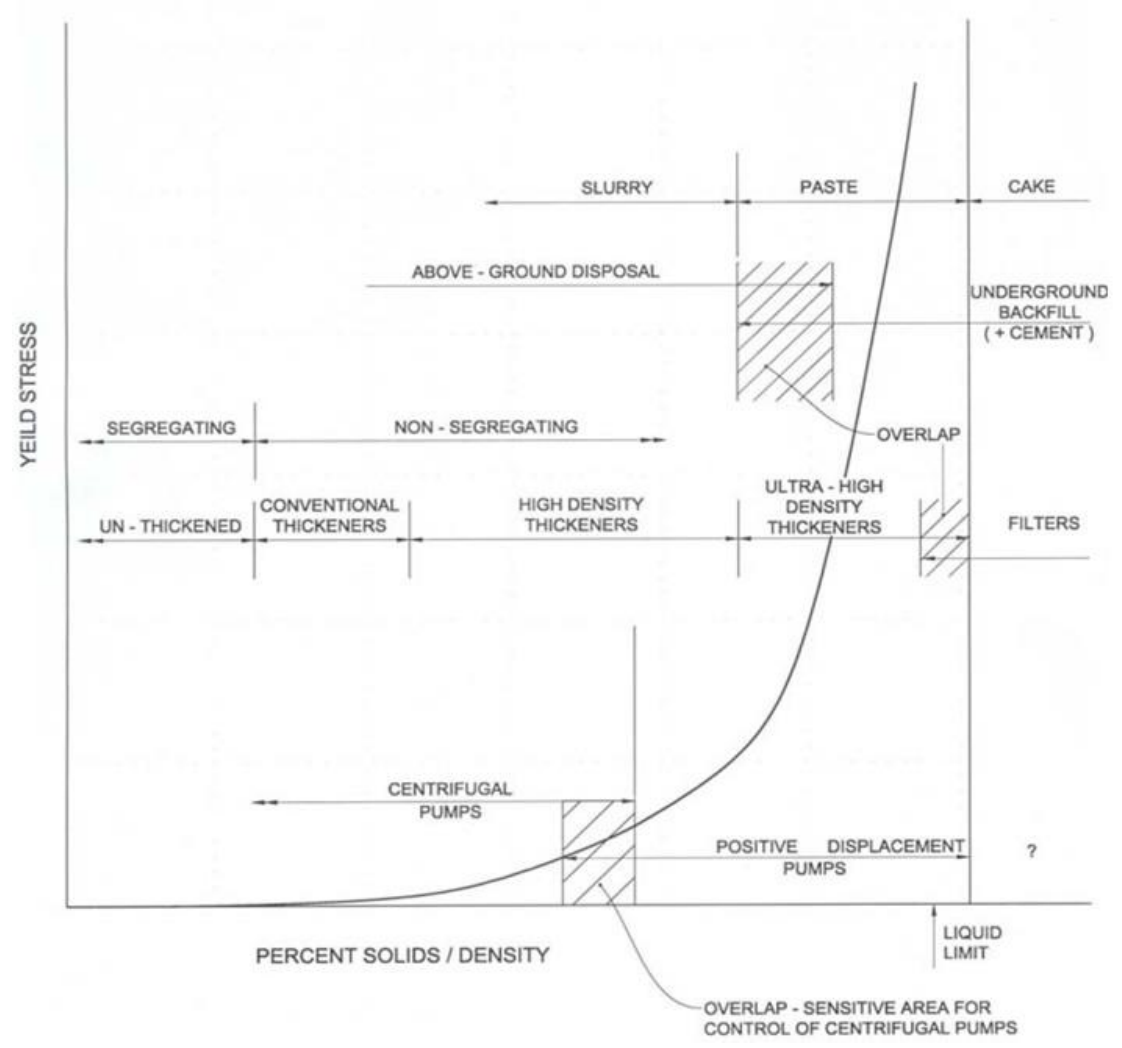

Figure 1 Paste and thickened tailings - indicative ranges of properties for different purposes (Jewell and Fourie, 2006) 
The curve in Figure 1 represents a plot of strength (say yield or shear stress) of a sample of tailings as the concentration of the sample (normally reported as solids content or density) is increased.

The action of removing water from the slurry at any point in the process and hence increasing the concentration of the solids essentially thickens and increases the strength of the material. From this, it can be seen that regardless of where one starts on the concentration axis, the removal of additional water further thickens the slurry. Hence, the process of thickening tailings may be viewed as a continuum. Within the continuum, however, it is possible to assign somewhat arbitrary labels such as slurry, paste and cake that describe the consistency of the product.

Paste thickening is simply the art of driving the thickening process closer to the theoretical minimum limit of water in the thickened underflow. The process itself is not new; many existing thickeners have been known to produce paste. Unfortunately, this generally occurs right before the thickener bogs and the rakes stop. The challenge is not the formation of paste, but the design of thickeners capable of generating paste in a controlled and continuous manner.

In the design of any thickener, whether it is a conventional high rate or paste unit, solids loading rate per unit of thickening area (solids flux rate in $t / \mathrm{m}^{2} \mathrm{hr}$ ) is a very important factor.

When the tailings have a high portion of sub $20 \mu \mathrm{m}$ material the solids loading rate decreases as does the achievable underflow density (Figure 2).

In a steady state thickening process, densification of the slurry can only proceed if the rate of water release from the slurry migrating upwards through the thickener towards the overflow is greater than the transport of the densified slurry towards the underflow. The rate of water release is governed by the natural permeability of the slurry and is enhanced by mechanical rake action.

Compression of the bed will provide an increase in underflow density whilst the compression zone remains sufficiently permeable for water to rise faster than the bed is falling through underflow withdrawal. Once the bed is compressed beyond this point, additional bed depth may actually hinder the release of water and limit the underflow density.

The time solids spend in the total bed does not govern the density achieved, only the time available for release of the water from the bottom of the bed.

The thickener must be designed to combine the effects of bed compression and solids flux rate to optimise the underflow density.

In addition, a number of studies have found that rake mechanisms that create channels in the thickener bed can assist with water release. Close attention must also be paid to the torque rating of the drive and mechanisms to prevent the formation of thick 'donuts' in the bed, which will reduce underflow density by hindering water release from the bed and potentially bogging the rakes. 


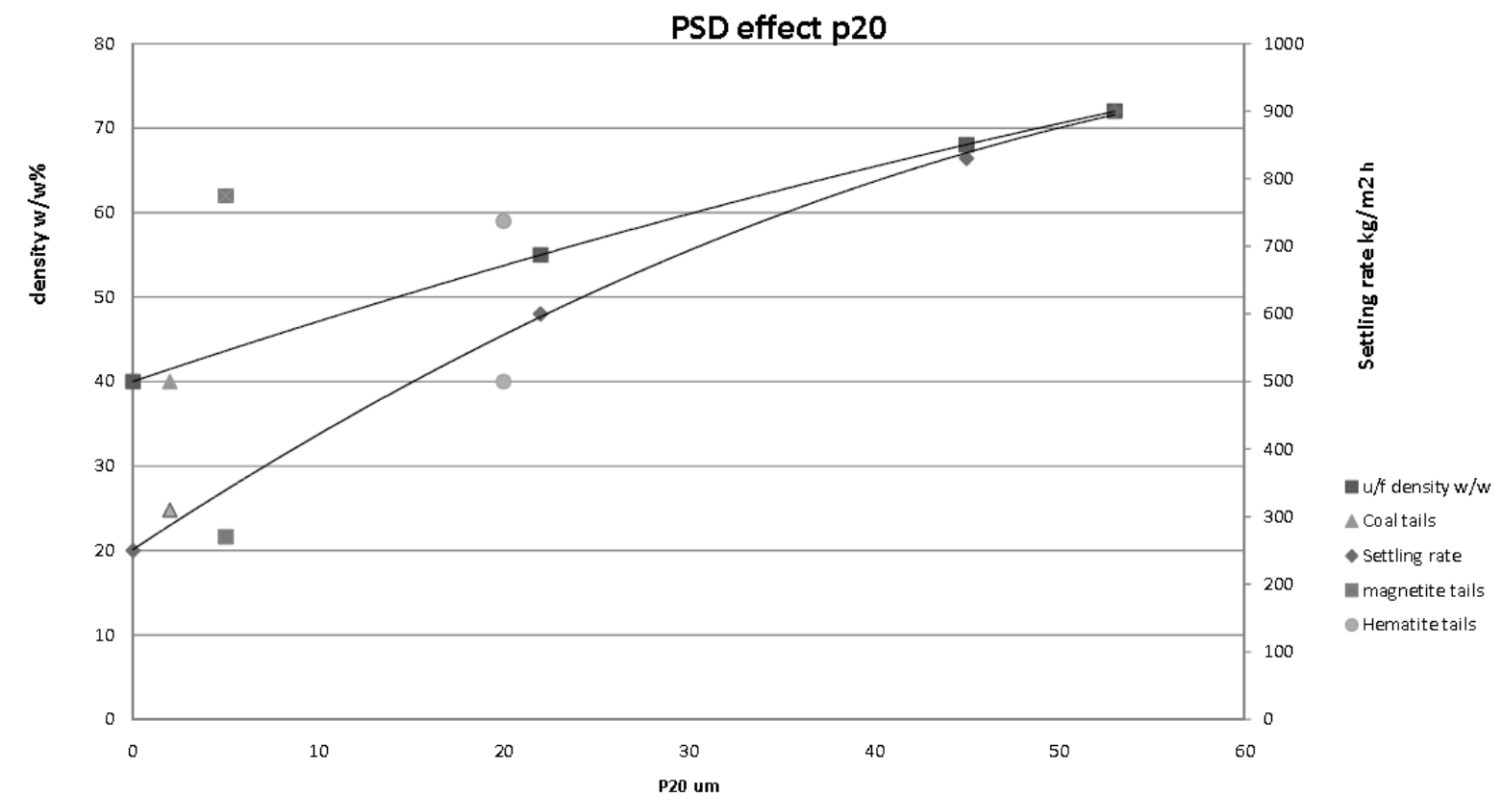

Figure 2 P20 effect on conventional sedimentation

\subsection{Vacuum filtration}

The two major variables in filtration are the void fraction, which determines the saturated moisture and the pore size, which limits the degree of moisture removal and defines the resistance to flow of water passing through the cake.

The driving force in vacuum filtration is atmospheric pressure and while liquid flow is co-current rather than counter current in sedimentation. The lack of mechanical compression means that the degree of separation possible without gas displacement is similar in both technologies. This maximum settled limit is similar to the shrinking limit observed in impoundment management. The limit depends upon the nature of particle stacking in the cake and the type of solids but the typical values for a ground mineral are in the range of $50-60 \%$ void volume.

Vacuum filtration has the advantage of solids handling capability whereas sedimentation would have long passed the pumpable limit that would enable solids removal from the sedimentation equipment.

For vacuum filtration to improve water recovery beyond the limits of sedimentation gas must flow into the void of the cake displacing water that can be recovered as filtrate. The nature of the void space in a cake can be best described as pores or channels passing between the solid particles.

When the tailings have a low portion of sub $20 \mu \mathrm{m}$ material, low cake moistures can be achieved and filtration capacities in the range of $1,000-5,000 \mathrm{~kg} / \mathrm{m}^{2} \mathrm{~h}$ are achievable (Figure 3).

When tailings contain a significant amount of fine material the capacity decreases rapidly and capacities can drop to below $100 \mathrm{~kg} / \mathrm{m}^{2} \mathrm{~h}$. 


\section{Tailings filtration HVBF}

Various tails

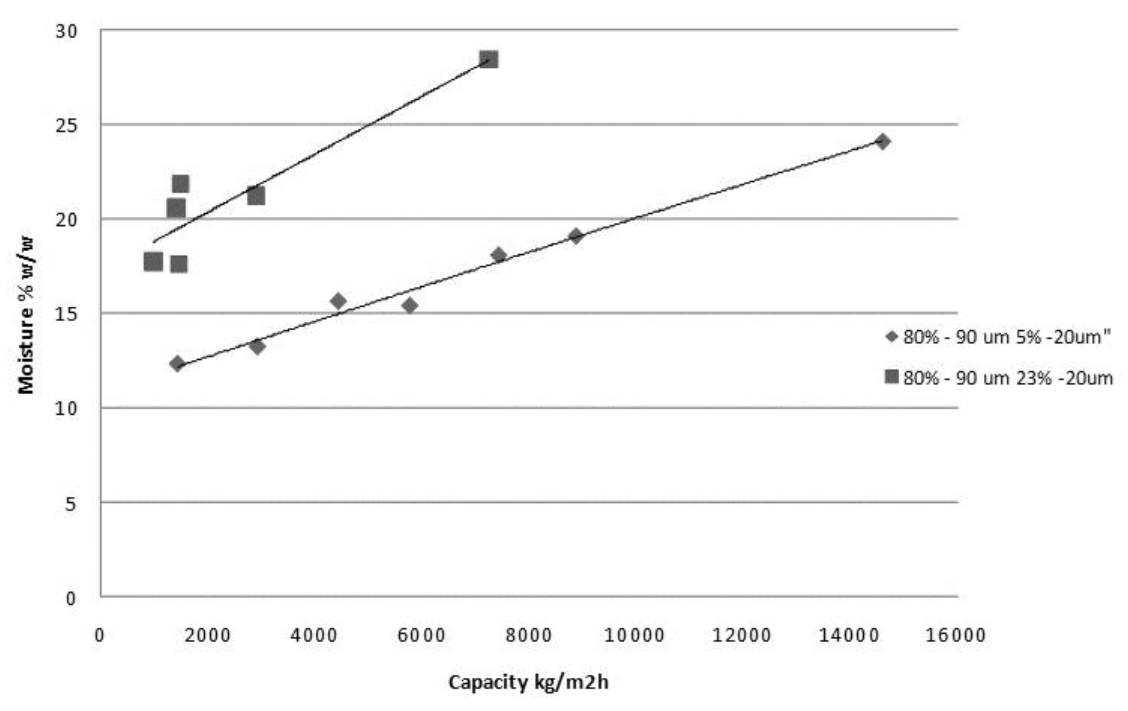

Figure 3 Vacuum filtration (horizontal vacuum belt filter (HVBF)) of various tails streams

\subsection{Pressure filtration}

Similar variables exist in pressure filtration. The driving force for pressure filtration is significantly higher and employment of mechanical compression means that a higher degree of water removal can be achieved. Here the void fraction is reduced and the typical range is from $45-55 \%$ void volume. In cases of ultra fine particles and high clay contents this can decrease to $35 \%$ void volume.

Pressure filtration with the limitation of fixed volume chambers and technical time associated with batch filtration does not achieve the very high capacities that are possible with vacuum filters. Using conventional fast opening double sided membrane filter presses (Figure 4) can achieve capacities of between $400-600 \mathrm{~kg} / \mathrm{m}^{2} \mathrm{~h}$ with easily dewatered material.

Where there is a large portion of clays or the particle size is small the filterability decreases and achievable moisture increases. Under these conditions the filtration rate may fall to below $100 \mathrm{~kg} / \mathrm{m}^{2} \mathrm{~h}$.

Tailings filtration FFP

tails

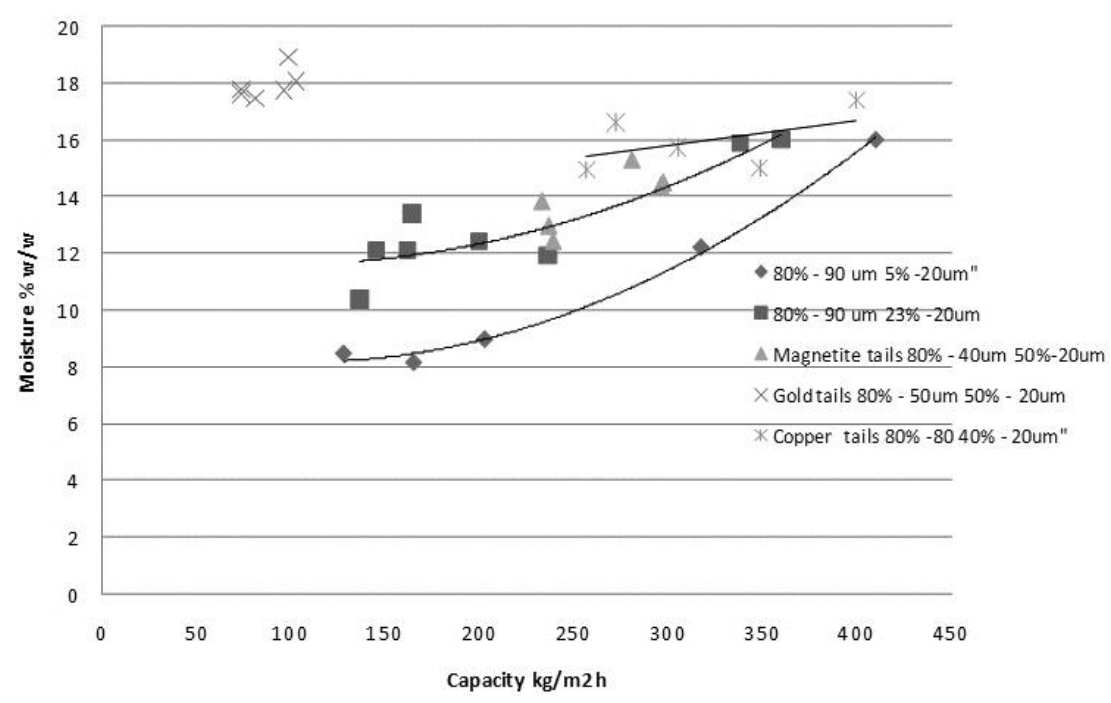

Figure 4 Pressure filtration (fast (opening) filter press (FFP)) of various tailings streams 


\section{$3 \quad$ Slurry particle properties}

\subsection{Void fraction}

The void fraction of either a cake or slurry is a measure of how tightly the solids are packed together. Where the cake is saturated this void will be filled with water and hence determines the moisture content of the mixture.

$$
\varepsilon=\frac{\left(V_{\text {cake }}-V_{\text {solids }}\right)}{V_{\text {cake }}}
$$

where:

$\begin{array}{lll}\varepsilon & = & \text { void fraction. } \\ V_{\text {solids }} & = & \text { Volume of solids }\left(\mathrm{m}^{3}\right) . \\ V_{\text {cake }} & = & \text { Volume of cake }\left(\mathrm{m}^{3}\right) .\end{array}$

Depending upon the type of array pure spheres can be packed with a void fraction of $0.26-0.77$ and with a cubic array giving a void fraction of 0.48 . During sedimentation the particles tend to form a loose packing.

Various strategies can be applied to increase the packing density but the most effective are shear and flocculants. Shear tends to shift particles sideways into valleys for a tighter stacking while coagulants and flocculants pull the particles together. As this packing density increases so too does the resistance to shear and the mixture becomes less fluid.

During filtration a similar packing begins but the co current flow of liquid tends to carry smaller particles through the cake filling voids and increasing the packing density. In pressure filtration mechanical force applied compresses the cake forcing particles to shift to higher density packing configurations.

\subsection{Particle size}

Sedimentation rates tend to be defined by the finest fraction unless flocculants are used, whereas the achievable underflow density is better suited to wider size distributions and with paste coarser fractions allow higher underflow densities.

Filtration technologies are best suited to tighter size distributions and slurries with a lower fraction of sub $20 \mu \mathrm{m}$ material. This low sensitivity can be best understood by looking at the separation that takes place during filtration (Figure 5). To achieve dry cakes it is necessary not only to form a cake but also to displace the liquid from the pores in the cake by using gas flow displacement. At small diameters the liquid in the pores is affected by surface tension at the gas liquid interface and a certain pressure is required to overcome the capillary forces.

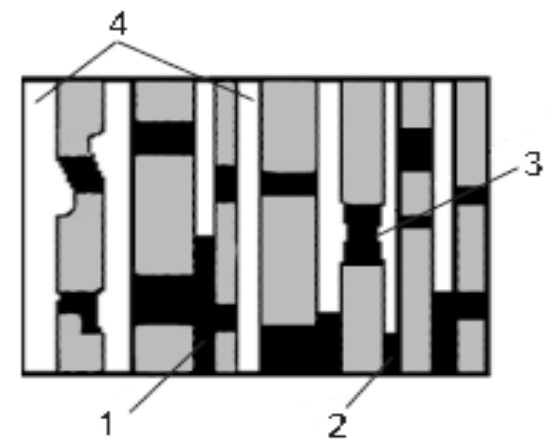

The structure of incompressible residue and methods for retaining the residual moisture.

1) local zones; 2) capillary moisture; 3) structured moisture; 4) working channels

Figure 5 Capillary pressure and gas displacement (Beloglazov et al., 2008) 
For air to enter the pores the differential pressure must exceed the capillary pressure. This pressure as shown in Equation (2) is a function of the pore diameter $D$, surface tension $\tau$ and the solid wetting angle $\theta$. Unlike flotation concentrates tailings generally have a hydrophilic surface and a large wetting angle that increases the threshold pressure (Wakeman and Tarleton, 2005).

When considering gas flow displacement then the relative permeability models of Lloyd and Dobbs (in Osborne, 1981) can be applied. This model can be used to predict the threshold pressure required for airflow.

$$
\begin{gathered}
\Delta P=\frac{4 \tau \cos \theta}{D} \\
P_{b}=\frac{4.6(1-\varepsilon) \sigma}{\varepsilon d}
\end{gathered}
$$

Where:

$\begin{array}{lll}P_{b} & = & \text { threshold pressure. } \\ \varepsilon & = & \text { void fraction. } \\ \sigma & = & \text { solids volume fraction. } \\ d & = & \text { particle diameter. } \\ \Delta P & = & \text { differential pressure. } \\ \theta & = & \text { wetting angle. } \\ \tau & = & \text { surface tension. } \\ D & = & \text { pore diameter. }\end{array}$

Putting these relationships into context for vacuum filters to be effective for dewatering of leach residues with a typical void fraction of $50 \%$ and differential pressure of 0.8 Bar, vacuum filter possibilities should extend to a mean particle size in the range of $25-30 \mu \mathrm{m}$.

Pressure filters remain a possibility for dewatering difficult slurries and here with a similar void fraction of $50 \%$ and differential pressure of $10 \mathrm{Bar}$, pressure filter possibilities should extend to a mean particle size in the range of $2.5 \mu \mathrm{m}$.

\section{PSD effect p20}

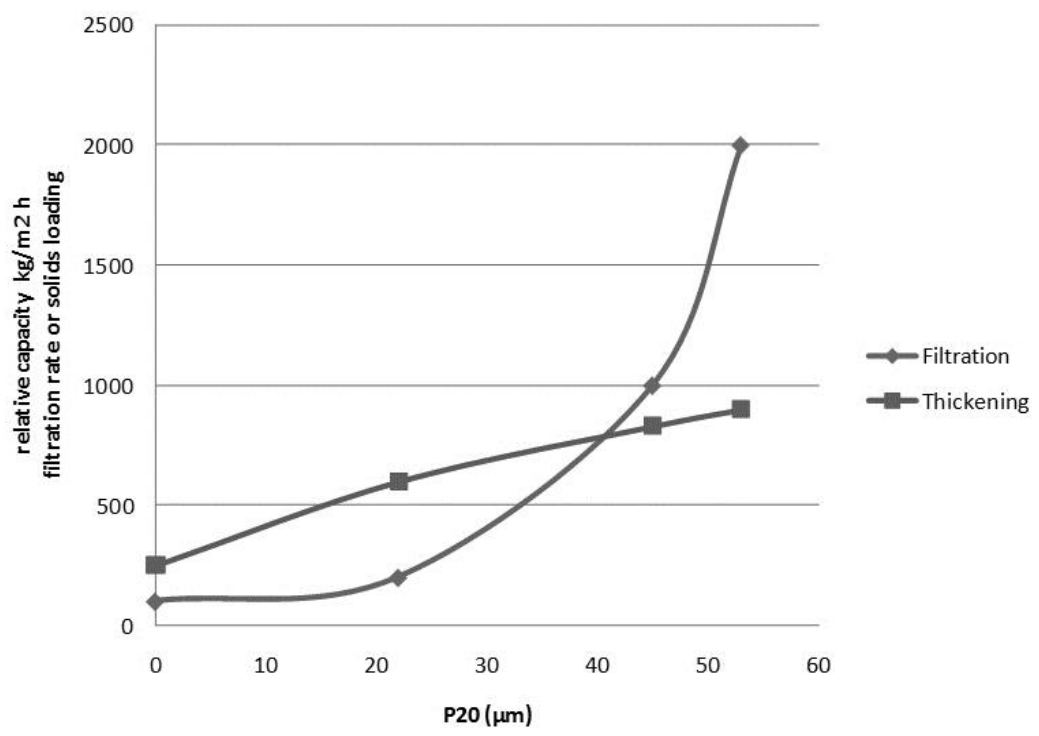

Figure 6 Filtration rate and thickener solids loading versus particle size 


\section{Dewatering options}

Most studies have concentrated on the option of paste or filtration combining all of the wastes to a single stream.

Filters are generally poor at dewatering dilute streams and increasing the filter feed density can significantly improve filtration performance. Belt filters in particular can be fed with paste where only a slight reduction in moisture may transform the material to a conveyable and stackable product.

With a typical mixed stream of tailings and paste thickening to $70 \% \mathrm{w} / \mathrm{w}$ underflow a belt filter could achieve $500 \mathrm{~kg} / \mathrm{m}^{2} \mathrm{~h}$ to dewater the stream to $80 \%$ solids provided there is a moderate to low fraction of sub $20 \mu \mathrm{m}$ material. Expanding the scope to produce cake would double the capital expense (CAPEX) of a paste only plant; however, the materials handling and impoundment will be simplified and would provide significant advantages over paste pumping systems and tailings dam impoundment.

Classification of a large tailings stream adds some complexity and cost but many processing plants have several different streams that with minimal flow sheet modifications could be separated to coarse and fine tailings streams. Dewatering the coarse $50 \%$ of the tailings with filtration and the remaining $50 \%$ with paste thickening technologies can provide very high water recoveries and substantial advantages in possible impoundment strategies.

When coarse tailings dewatering rates exceed $1,500 \mathrm{~kg} / \mathrm{m}^{2} \mathrm{~h}$ (Figure 6) the cost of filtration may be lower than sedimentation technologies and provides valuable material for impoundment wall construction. Depending upon the results achievable combining paste and dry cake may still achieve the dry stackable requirements with the added advantages of high water recovery and disposal costs.

Where tailings streams contain significant amounts of clays and ultra fine fraction solids conventional and paste thickening technologies will continue to be the most attractive option. At present the thickening technologies are determined by the available slurry transport options.

In some cases where dry stacking of difficult slurries is required increasing the $\mathrm{u} / \mathrm{f}$ density up to the limit pumpable by centrifugal pumps will enhance the performance of pressure filtration. Paste sedimentation technologies hence have a strong advantage over conventional thickening and will significantly reduce the filtration costs.

\section{Conclusions}

Most studies have concentrated on the option of paste or filtration combining all of the wastes to a single stream. Improvements to current practise may be achieved by studying splitting of process tailings streams to better suit the strengths of the differing separation technologies rather than forcing one technology to handle all that is produced. Classification of a large tailings stream adds some complexity and cost but many processing plants have several different streams that, with minimal flow sheet modifications could be separated to coarse and fine tailings streams. Dewatering the coarse $50 \%$ of the tailings with filtration and the remaining $50 \%$ with paste thickening technologies can provide very high water recoveries and substantial advantages in possible impoundment strategies. Depending upon the results, combining paste and dry cake may still achieve the dry stackable requirements with the added advantages of high water recovery and disposal costs. Where tailings streams contain significant amounts of clays and ultra fine fraction solids, paste thickening technologies will continue to be the most attractive option. Cases where dry stacking of difficult slurries is required, increasing the underflow density up to the pumpable limit for centrifugal pumps will enhance the performance of pressure filtration.

\section{Acknowledgements}

The assistance of Outotec Oyj in preparation and permission to publish is acknowledged.

\section{References}

Beloglazov, I.N., Golubev, V.O., Tikhonov, O.N., Mikheyev, A.I., Beloglazov, I.I., Vorobyov, S.A., Palmer, J., Kuukka, J., Jaaskelainen, E. and Rogov, S.N. (2008) Filtration of Process fine materials, Ore and Metals, Russia. 
Jewell, R.J. and Fourie, A.B. (eds) (2006) Paste and Thickened Tailings - A Guide, 2nd edition, Australian Centre for Geomechanics, Perth, 242 p.

Osborne, D.G. (1981) Vacuum Filtration - Part I, in Solid-Liquid Separation, 2nd Edition, L. Svarovsky (ed), Butterworth \& Co Ltd: London, p. 349.

Wakeman, R.J. and Tarleton, E.S. (2005) Solid/liquid separation: principles of industrial filtration, Elsevier, Oxford, UK. 